ache. Intellectually unfit to grapple with these subtilties, I must return to the simple, if crude and ignorant, physiological conception that all characters are products of germinal predisposition and somatic nurture; and to the notion that, while there is always blending and sometimes alternate reproduction, there is never alternate inheritance.

20 Lennox Road, South, Southsea, Hants,

$$
\text { G. Archdall Reid. }
$$

$$
\text { May } 17 .
$$

\section{Martini's Equations for the Epidemiology of Immunising Diseases.}

THE differential equations constructed by Dr. Martini and quoted by Dr. Lotka in NATuRE of May I2, p. 633, namely,

$$
\begin{aligned}
& \frac{d u}{d t}=a u(\mathrm{x}-i)-q u, \\
& \frac{d i}{d t}=a u(\mathrm{x}-i)-m u,
\end{aligned}
$$

aroused my interest by the statement that they cannot be integrated in finite terms. I have noticed that in one particular case the integrals of the equations can be expressed in a moderately simple form, and that in this case Dr. Martini's second position of equilibrium, namely,

$$
u=\frac{m(a-q)}{a q}, \quad i=\frac{a-q}{a},
$$

is unattainable within a finite time unless it is permanent.

The particular case in question which has come to my notice is that in which $q=m$, that is to say, the fraction of the affected population which ceases to be so per unit time is equal to the fraction of the immune population which loses immunity or dies per unit time.

In this case it is evident by subtraction that

$$
\frac{d u}{d t}-\frac{d i}{d t}=-q(u-i), ?
$$

so that $u-i=\mathrm{A} q e^{-q t}$, where $\mathrm{A}$ is the constant of integration. On substituting for $i$ in (I) it is evident that

$$
\frac{d u}{d t}=\left(a-q+a \mathrm{~A} q e^{-q t}\right) u-\alpha u^{2},
$$

an equation reducible to the linear form (and so soluble by quadratures) by the substitution $v=\mathrm{I} / u$.

The solution, which it will be sufficient to quote, is

$$
\begin{aligned}
\frac{\mathrm{I}}{u}=\sum_{n=0}^{\infty} \frac{(-)^{n} a^{n+1} \mathrm{~A}^{n} e^{-n q t}}{(a-q)(a-2 q) \cdots(a-n q-q)}, & \\
& +\mathrm{B} \exp \left\{(q-a) t+a \mathrm{~A} e^{-q t}\right\},
\end{aligned}
$$

where $B$ is the second constant of integration.

It is easy to see that the second position of equilibrium is given in this case by

$$
u=i=\frac{a-q}{a},
$$

and if $u$ and $i$ have this value for a finite value of $t$, it is readily seen successively that $\mathrm{A}=0$ and $\mathrm{B}=0$, so that $u$ and $i$ have this value for all time. On the other hand, whatever be the values of $\mathrm{A}$ and $\mathrm{B}$, the value $(a-q) / a$ is the limit to which both $u$ and $i$ tend as the time tends to infinity, provided that $a$ exceeds $q$; if a does not exceed $q$, they tend to zero, unless $\mathrm{B}=\mathrm{o}$.

I imagine from Dr. Lotka's silence concerning these results that they have not been previously obtained, and, for all I know, the case $q=m$ may be of no practical importance. But the analysis which I have given seems to me to throw some light on what the behaviour of the solution might be expected to be in the general case.

The University, Birmingham, May I2.

G. N. Watson.

\section{The Structure of Basic Beryllium Acetate.}

THE remarkable compound $\mathrm{Be}_{4} \mathrm{O}\left(\mathrm{C}_{2} \mathrm{H}_{3} \mathrm{O}_{2}\right)_{6}$, the crystal structure of which Sir William Bragg describes in his letter in NATURE of April 2x, p. 532, can be given a chemical formula in complete accordance with its properties. Tanatar and Kurowski have described (Journ. Russ. Phys.-Chem. Ges. 39, 936, I630; 40, 787 ; Chem. Centr. I908, I. I02, I523; II. I409) a series of compounds (including the formate, acetate, propionate, and benzoate) of the general formula $\mathrm{Be}_{4} \mathrm{OA}_{6}$. These compounds have none of the characteristics of salts. They are volatile, they have low melting-points (some are liquid), they are soluble in organic solvents such as benzene, and they do not conduct electricity.

They resemble the non-ionised members of the "chelate" series of compounds, to which Prof. Morgan has directed attention. These are derived from substances containing such groupings as $\mathrm{HO}-\mathrm{C} \ldots \ldots \mathrm{C}=\mathrm{O}$, which combine with an atom of a metal by replacement of the hydroxyl hydrogen, and at the same time also (as he has shown) through the carbonyl oxygen. The simplest example is the volatile beryllium acetylacetonate (formula I.). The carbonyl oxygens becoming trivalent must each lose an electron. These two electrons go to the beryllium, which already has two valency electrons, and thus give it the four required to constitute the four nonpolar links in the resulting compound. Chelate compounds of this type, in which the group is attached through both oxygens to the same metallic atom, are not formed by the carboxyl group, obviously because this would lead to the formation of a 4-ring, which is unstable. But there is no reason why the carboxyl should not react in this way if the attachment is to two different metallic atoms, with the formation of a ring of 5 or 6 atoms.

This must happen with basic beryllium acetate. We have at the centre, as Sir William Bragg suggests, the oxygen atom attached to 4 beryllium atoms. The octet of the oxygen is made up of 4 electrons from the four beryllium atoms, and four from the oxygen. But the oxygen atom originally had six valency electrons, and so it must lose two. The attachment of the acetate group to two beryllium atoms is shown in formula II. It forms a 6-ring :-

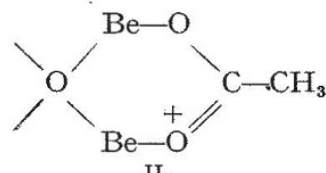

II.<smiles>CC1=CC(C)=[O+][B-]2(O1)OC(C)=CC2C</smiles>
I.
But in forming the ring each acetate group must lose an electron from its carbonyl oxygen, so that the six give up six electrons, in addition to the two given up by the central oxygen. We therefore have eight electrons, two of which go to each beryllium atom, increasing its valency electrons from 2 to 4 . Thus each beryllium atom can form four non-polar links, these being (I) to the central oxygen, and $(2,3,4)$ through three acetate groups to each of the other three beryllium atoms. One of these six chelate groups thus corresponds to each edge of the tetrahedron. 